\title{
Dopant Migration-Induced Interface Dipole Effect in n-Doped GaAs/AlGaAs Terahertz Detectors
}

\author{
Satyabrata Jit, Aruna Bandara Weerasekara, Ranga Chaminda Jayasinghe, Steven G. Matsik, \\ A. G. Unil Perera, Margaret Buchanan, G. Irwin Sproule, H. C. Liu, Andreas Stintz, \\ Sanjay Krishna, S. P. Khanna, M. Lachab, and E. H. Linfield
}

\begin{abstract}
A heterojunction interfacial workfunction internal photoemission (HEIWIP) terahertz detector with $\sim 1 \times 10^{18} \mathrm{~cm}^{-3}$ n-type doped GaAs emitters in a multilayer $\mathrm{GaAs} / \mathrm{Al}_{0.13} \mathrm{Ga}_{0.87} \mathrm{As}$ heterostructure is presented. The detection mechanism is based on free carrier absorption with a broad response extending to $\sim 5.26 \mathrm{THz}(57 \mu \mathrm{m})$, corresponding to an effective workfunction of $\sim 21.8 \mathrm{meV}$, which is much smaller than the offset expected for an Al fraction of $x=0.13$ at a $1 \times 10^{18} \mathrm{~cm}^{-3}$ doping. This is attributed to a reduction of the conduction band offset by interface dipole formation between the accumulated negative charges at the interface states and migrated positively charged donors in the barrier. The device has a peak responsivity of $0.32 \mathrm{~A} / \mathrm{W}$ at $\sim 26 \mu \mathrm{m}$ at $5 \mathrm{~K}$. It is demonstrated that the dopant migration-induced interface dipole effect can be used to extend the zero response threshold frequency $\left(f_{0}\right)$ of n-type HEIWIP detectors.
\end{abstract}

Index Terms-Dopant migration, GaAs/AlGaAs, heterojunction, infrared detectors, terahertz (THz) detectors.

$\mathbf{T}$ ERAHERTZ (THz) photodetectors have drawn considerable attention due to their potential applications in areas such as medical diagnostics, pharmaceutical, security, wireless communication, and astronomy. Conventional photodetectors such as MCT, QWIP, etc., have threshold frequencies above $10 \mathrm{THz}(30 \mu \mathrm{m})$. The competitive approaches for THz detection include homojunction [1] and heterojunction interfacial workfunction internal photoemission (HEIWIP) [2] THz detectors in which the basic detection mechanism is the free carrier absorption in an emitter region and the collection of the photoexcited

Manuscript received April 8, 2008. First published September 9, 2008; current version published September 24, 2008. This work was supported in part by the U.S. National Science Foundation under Grant ECS-0553051. The review of this letter was arranged by Editor G. Meneghesso.

S. Jit is with the Department of Physics and Astronomy, Georgia State University, Atlanta, GA 30303-4106 USA and also with the Department of Electronics Engineering, Institute of Technology, Banaras Hindu University, Varanasi 221005, India (e-mail: sjit.ece@itbhu.ac.in).

A. B. Weerasekara, R. C. Jayasinghe, S. G. Matsik, and A. G. U. Perera are with the Department of Physics and Astronomy, Georgia State University, Atlanta, GA 30303-4106 USA (e-mail: aweerasekara3@mail.gatech.edu; rjayasinghe@student.gsu.edu; smatsik@gsu.edu; uperera@phy-astr.gsu.edu).

M. Buchanan, G. I. Sproule, and H. C. Liu are with the Institute for Microstructural Sciences, National Research Council, Ottawa, ON K1A 0R6, Canada (e-mail: margaret.buchanan@nrc-cnrc.gc.ca; irwin.sproule@nrc-cnrc. gc.ca; H.C.Liu @ nrc-cnrc.gc.ca).

A. Stintz and S. Krishna are with the Department Electrical Engineering and Computer Engineering, Center for High Technology Materials, University of New Mexico, Albuquerque, NM 87106 USA (e-mail: andreas@ chtm.unm.edu; skrishna@chtm.unm.edu).

S. P. Khanna, M. Lachab, and E. H. Linfield are with the School of Electronic and Electrical Engineering, University of Leeds, LS2 9JT Leeds, U.K. (e-mail: s.p.khanna03@leeds.ac.uk; m.lachab@leeds.ac.uk; e.h.linfield@leeds.ac.uk).

Digital Object Identifier 10.1109/LED.2008.2002946 carriers over an undoped barrier. Most HEIWIP detectors [2] reported are p-type doped emitters. Faster response speed due to low effective carrier mass in n-type HEIWIP detectors may be of interests for high-speed applications. In this letter, the effect of dipole formation due to dopant migration on the threshold frequency $\left(f_{0}\right)$ of n-type GaAs/AIGaAs HEIWIP THz detector is explored. Using the dipole concept, the barrier height of n-type GaAs/AlGaAs HEIWIP detectors can be lowered making the $\mathrm{THz}$ detection possible.

The schematic diagram of the GaAs/ $\mathrm{Al}_{0.13} \mathrm{Ga}_{0.87} \mathrm{As}$ multilayer HEIWIP detector under consideration is shown in Fig. 1(a). The device consists of 12 periods of $1 \times 10^{18} \mathrm{~cm}^{-3}$ Si-doped $20 \mathrm{~nm}$ GaAs emitter and undoped $80 \mathrm{~nm}$ $\mathrm{Al}_{0.13} \mathrm{Ga}_{0.87} \mathrm{As}$ barrier, sandwiched between two $1 \times 10^{18} \mathrm{~cm}^{-3}$ $\mathrm{Si}$-doped GaAs contact layers. The structure was grown by molecular beam epitaxy and GaAs growth rate was $0.9 \mathrm{ML} / \mathrm{s}$ while AlGaAs growth rate was $1.0 \mathrm{ML} / \mathrm{s}$. A 200-nm-doped GaAs buffer was grown at $580{ }^{\circ} \mathrm{C}$, and the rest of the structure was grown at $640{ }^{\circ} \mathrm{C}$. As to $\mathrm{Ga}$, beam equivalent pressure ratio was $10: 1$. The $\mathrm{n}$-doped GaAs substrate enhances the light reflection improving the light absorption in emitter regions. The raw spectral response of the detector was measured using a Fourier transform infrared spectrometer, and a Si composite bolometer was used to normalize the raw spectral response. Using the bolometer sensitivity $\left(3.5 \times 10^{5} \mathrm{~V} / \mathrm{W}\right)$ and circuit impedance, the detector spectral response was calculated in ampere/watts units.

The conduction band diagram of a single heterojunction under zero-bias condition is shown in Fig. 1(b). The workfunction $(\Delta)$ for an ideal heterojunction interface (i.e., zero interface states) is $\Delta=\Delta E_{C}-E_{F}=\Delta_{x}+\Delta_{d}-E_{F}$ as considered in an earlier HEIWIP detector [3]. Here, $\Delta E_{C}=\Delta_{d}+$ $\Delta_{x}$. The band offset, $\Delta_{x}=790 x$, is due to the $\mathrm{Al}$ fraction $x$ in the $\mathrm{Al}_{x} \mathrm{Ga}_{1-x} \mathrm{As}$ barrier, and $\Delta_{d}$ is the shift in the conduction band of the GaAs due to the doping. $E_{F}$ is the Fermi level with respect to the bottom of the conduction band of the emitter.

Following the conventional design methodology of the reported HEIWIPs [3], the present device should give $\Delta=\Delta E_{C}-E_{F} \approx 82.8 \mathrm{meV}$ resulting in $f_{0}(\mathrm{THz})=$ $\Delta(\mathrm{meV}) / 4.133 \approx 20 \mathrm{THz}$ under zero bias condition. The $(\Delta)$ can be estimated using the Arrhenius plot, where the slope of $\ln \left(\mathrm{I} / \mathrm{T}^{1.5}\right)$ versus $1 / \mathrm{T}$ is proportional to $\Delta$. Arrhenius analysis in Fig. 2(a) shows the measured $\Delta$ varing from $\sim 24.2$ to $20.7 \mathrm{meV}$ corresponding to bias voltage from -20 to $-180 \mathrm{mV}$, which is much smaller than the expected value of $82.8 \mathrm{meV}$. To investigate the discrepancy, secondary ion mass spectrometry 


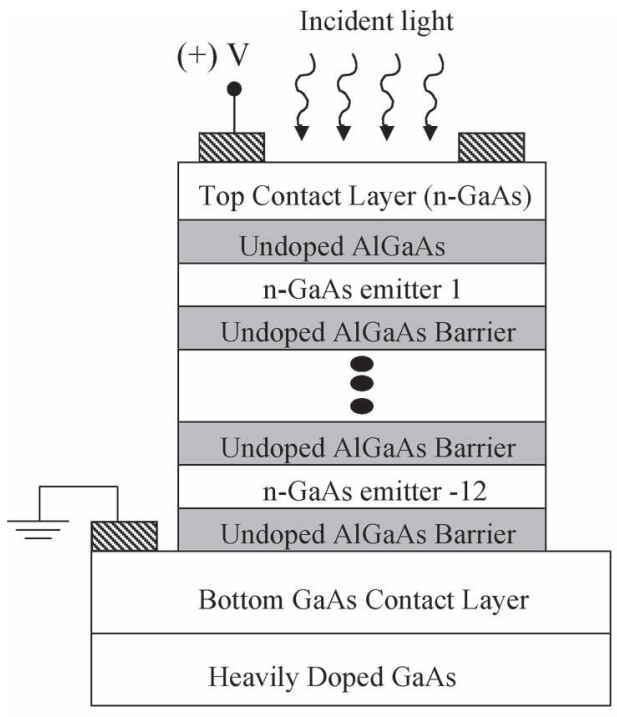

(a)

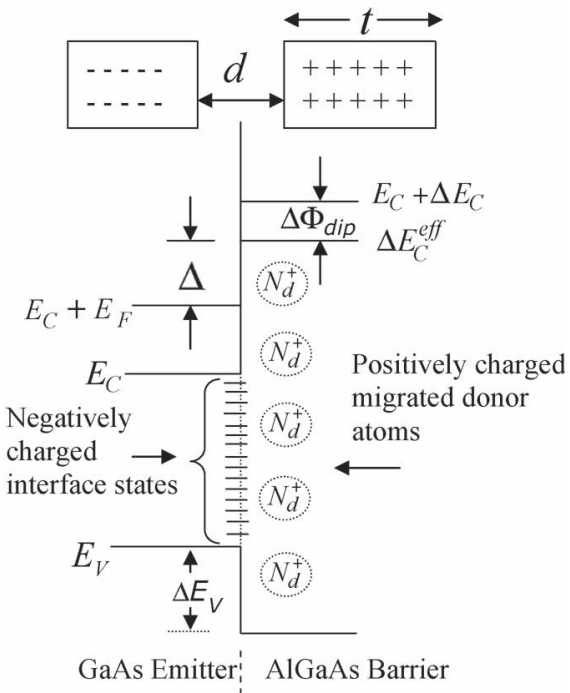

(b)

Fig. 1. (a) Schematic structure of the detector. The device structure parameters are given in the text. Forward bias-top contact $(+)$ ve. Reverse bias-top contact (-)ve. (b) Band diagram of an unbiased emitter illustrating the reduction in conduction band discontinuity due to interface dipole effect.
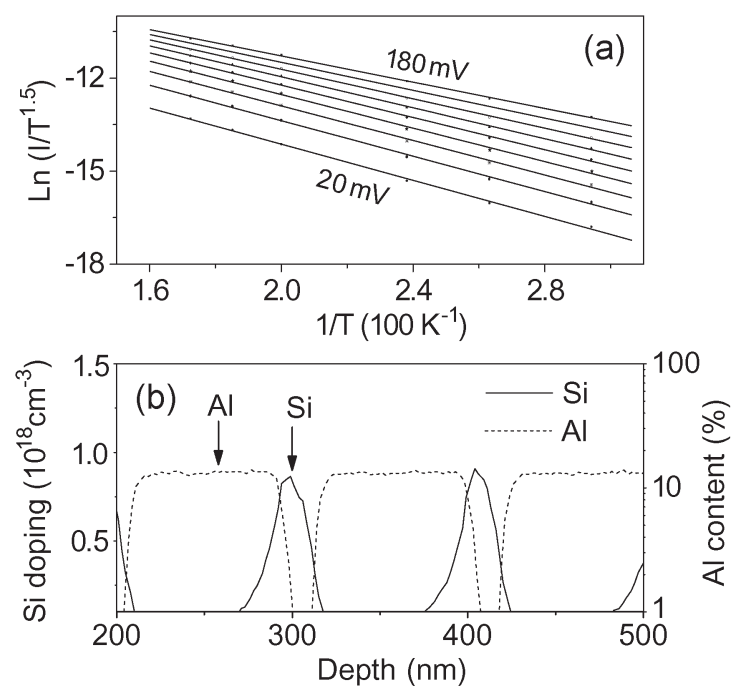

Fig. 2. (a) Arrhenius plot to estimate $\Delta$ at different applied bias voltages between 20 and $180 \mathrm{mV}$. (b) SIMS profile of a part of the GaAs/AlGaAs multilayer structure showing the dopant migration in the barrier region.

(SIMS) measurements [shown in Fig. 2(b)] were carried out which confirms $N_{d}=1 \times 10^{18} \mathrm{~cm}^{-3}$ and $x \sim 0.13$ used in the calculations. Shown in Fig. 2(b), a significant dopant migration with peak concentration $\sim 4 \times 10^{17} \mathrm{~cm}^{-3}$ is observed in the growth direction into the barrier region which decreased to nearly zero at $\sim 30 \mathrm{~nm}$ from the interface. The migrated donor atoms release their electrons and form a positively charged region in the barrier near the interface. The quality of the heterojunction interface may be deteriorated severely by heavily doped emitters [4], and the increased interface roughness may lead to enhancement in the interface state density at the heterointerface similar to results reported in [5]. The band discontinuity can significantly be affected [6] if a dipole layer is formed across a heterointerface. Rimmer et al. [7] found the accumulation of a negatively charged region of interface states at the GaAs/AlGaAs heterointerfaces agreeing with Kroemer's theory [8]. The origin of the interface states can be the dangling bonds in the interfaces, Coulomb potential of charged ions, and shallow impurities near interfaces [9]. The net negatively charged region at the heterointerface may include the accumulation of shallow donors [7]. However, the negatively charged interface states and migrated positively charged donor atoms may form an interface dipole [6] as shown in Fig. 1(b) that reduces the conduction band discontinuity. The interface dipole reduces ([6]) the conduction band offset $\Delta E_{C}$ by an amount $\Delta \Phi_{\text {dip }}(\mathrm{eV})=\left(\sigma / \varepsilon_{b}\right)(d+t)$, where $\sigma=q N_{b} t$ is the chargesheet density in the dipole, $d$ is the separation between the positive and negative charge regions, $t$ is the width of the positively charged region in the barrier, $q$ is the electronic charge, $\varepsilon_{b}$ is the permittivity of the barrier material, and $N_{b}$ is the average donor concentration in the barrier over a thickness of $t$. The average $N_{b}$ is estimated to be $\sim 4.6 \times 10^{16} \mathrm{~cm}^{-3}$ using the SIMS profile. Since the donor atoms extend over a $\sim 30 \mathrm{~nm}$ from the interface, and the negative charge region is very close to the heterointerface giving $t \gg d$, therefore, $\Delta \Phi_{\text {dip }} \approx\left(\sigma / \varepsilon_{b}\right) t=57.6 \mathrm{meV}$, and the effective conduction band offset becomes $\Delta E_{C}^{\mathrm{eff}} \approx \Delta E_{C}-q\left(\sigma / \varepsilon_{b}\right) t=80 \mathrm{meV}$. The interface state density is normally less than $10^{11} \mathrm{~cm}^{-2}$ which affects the band offset by less than $10 \mathrm{meV}$ in most of the GaAs/AlGaAs heterojunctions [10]. Since the heavy doping may increase interface roughness [4] increasing the interface state density [5] and resulting in the ionized donor density $\left(N_{b} t \sim 1.38 \times 10^{11} \mathrm{~cm}^{-2}\right)$ over a larger thickness $(\sim 30 \mathrm{~nm})$ than in normal heterointerfaces $(<10 \mathrm{~nm}$ near the interface $)$, the interface dipole can reduce $\Delta E_{C}$ significantly $(\sim 57.6 \mathrm{meV})$ in the present device. However, in the case of an intentionally introduced doping interface dipole in band offset engineering of a heterojunction [6], $t$ is maintained to be very small as compared to $d<100 \AA$ resulting in $\Delta \Phi_{\text {dip }}(\mathrm{eV}) \approx\left(\sigma / \varepsilon_{s}\right) d$. 


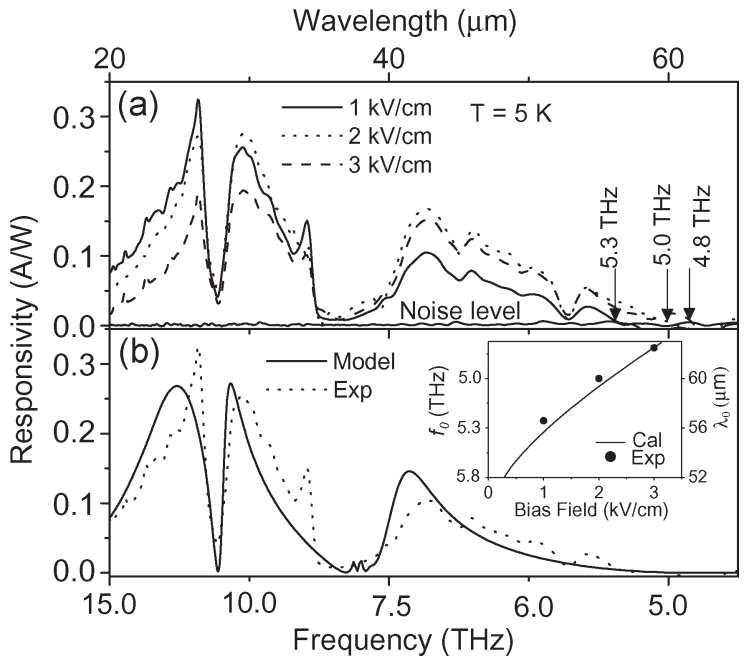

Fig. 3. (a) Measured photoresponsivity of the detector for different electric fields at $5 \mathrm{~K}$. The maximum responsivity $0.32 \mathrm{~A} / \mathrm{W}$ at $11 \mathrm{THz}$ at the field of $1 \mathrm{kV} / \mathrm{cm}$ is obtained. (b) Comparison of the theoretical and experimental photoresponsivities at $1 \mathrm{kV} / \mathrm{cm}$. The inset shows the comparison of the (solid line) calculated and (dots) experimental $f_{0}$ as a function of bias fields.

The bias dependence of $\Delta$ is due to the image force which reduces $\Delta$ by an amount $\Delta \phi=q \sqrt{q E_{b} / 4 \pi \varepsilon_{b}}$ giving $\Delta(\mathrm{meV})=$ $\Delta E_{C}^{\mathrm{eff}}-E_{F}=25.2-\Delta \phi(\mathrm{meV})$, where $E_{b} \approx V_{b} / 13 L_{b}$ is the field in the barrier, $V_{b}$ is the bias voltage, and $L_{b}$ is the barrier width. This gives $\Delta \sim 23.7$ and $20.8 \mathrm{meV}$ corresponding to their measured values of 24.2 and $20.7 \mathrm{meV}$ for bias voltages 20 and $180 \mathrm{mV}$, respectively.

The spectral responsivity for different fields is shown in Fig. 3(a). The broad responsivity spectrum, extending to $\sim 57 \mu \mathrm{m}$ (i.e., $5.2 \mathrm{THz}$ ) for a filed of $E_{b} \sim 1 \mathrm{kV} / \mathrm{cm}$, clearly demonstrates the free carrier absorption mechanism of the detector with a peak responsivity of $0.32 \mathrm{~A} / \mathrm{W}$ at $\sim 11.5 \mathrm{THz}$ $(26 \mu \mathrm{m})$ at $5 \mathrm{~K}$. Furthermore, the decrease in the $f_{0}$ with the field is also clearly visible. The experimental $f_{0}$ was determined with respect to the measured system noise level shown in the figure. The dips at $\sim 35$ and $\sim 27 \mu \mathrm{m}$ correspond to AlAs-like and GaAs phonons, respectively. The experimental result for $E_{b} \sim 1 \mathrm{kV} / \mathrm{cm}$ is compared to the calculated [3] responsivity in Fig. 3(b). Despite the similar characteristics, a magnitude discrepancy was found between the calculated and the measured spectra, and the calculated spectrum was scaled to match the experimental spectra. The little discrepancies between the two results may be attributed to the impurity scattering (due to dopant migration) in the barrier. A comparison of the experimental and calculated $f_{0}(\mathrm{THz})=\Delta(\mathrm{meV}) / 4.133$ as a function of the applied field $E_{b}$ is shown in the inset of Fig. 3(b) which shows a close agreement.

The lower responsivity compared to the single emitter-single barrier HEIWIP detector [3] may be attributed to the following reasons: 1) the lower absorption region $(\sim 12 \times 20 \mathrm{~nm}=$ $240 \mathrm{~nm}$ for 12 emitters) may result in $\sim 65 \%$ less photocurrent than that of [3] (with a 700-nm-thick emitter) due to the reduction in the quantum efficiency; 2) larger threshold barrier energy ( $\sim 25.2 \mathrm{meV}$ ) as compared to $\sim 13 \mathrm{meV}$ of [3] can cause less electrons to escape into the barrier and, thus, may result in smaller photocurrent; and 3) the possibility of escaped carriers from a particular emitter being captured by other emitters during their carrier transportation. However, the magnitude of charged interface state density in a heterointerface, in general, is highly sample-dependent (under the same growth conditions) [7]. Thus, it may be quite reasonable to assume that samples grown under different growth conditions should have different interface state densities. This may be one of the possible reasons for not observing interface dipole effect in single emitter device [3] with less dopant migration $\left(\sim 9 \times 10^{17} \mathrm{~cm}^{-3}\right)$ over thinner region $(\sim 5 \mathrm{~nm})$. A second device (L200) with 12 periods of $1.3 \times 10^{18} \mathrm{~cm}^{-3}$ Si-doped 20-nm GaAs emitter and undoped 80-nm $\mathrm{Al}_{0.09} \mathrm{Ga}_{0.91} \mathrm{As}$ barrier was tested and shows $f_{0} \sim 4 \mathrm{THz}(\sim 70 \mu \mathrm{m})$ in forward bias operation and $\sim 9 \mathrm{THz}(\sim 34 \mu \mathrm{m})$ in the reverse bias operation, which can be a consequence of the dipole effect. According to the SIMS measurements in the second device, the migrated dopant concentration starting from $\sim 4 \times 10^{17} \mathrm{~cm}^{-3}$ on the GaAs/AlGaAs interface reaches $\sim 1 \times 10^{16} \mathrm{~cm}^{-3}$ over a thickness of $15 \mathrm{~nm}$ in the growth direction giving $\Delta \Phi_{\mathrm{dip}} \approx\left(\sigma / \varepsilon_{b}\right) t \sim 24 \mathrm{meV}$. Without dipole effect, the calculated $\Delta$ is $\sim 40 \mathrm{meV}$ giving $f_{0} \sim 10 \mathrm{THz}(30 \mu \mathrm{m})$ which roughly matches with observed $f_{0}$ in reverse bias. With the dipole effect, the calculated $\Delta$ would be $\sim 16 \mathrm{meV}$ resulting in $f_{0} \sim 3.9 \mathrm{THz}(77 \mu \mathrm{m})$ which matches with the observed $f_{0}$ in forward bias. The $\mathrm{Al}$ fraction in the above structure was determined by XRD measurements and numerical fittings. In case of p-type HEIWIP [2], no dopant migration was observed in the reported detectors since there is no possibility of formation of an interface dipole between interface states and ionized acceptor atoms because of their identical charge polarity. Note that the dipole effect is not observed in multiple quantum well devices due to the $\delta$ doping method, which cannot be used for free carrier absorption-based detectors.

To conclude, the effect of interface dipole due to dopant migration in the barrier region of a Si-doped $\mathrm{GaAs} / \mathrm{Al}_{0.13} \mathrm{Ga}_{0.87} \mathrm{As}$ multilayer HEIWIP THz detector is presented. This letter shows that, dopant migration in n-type HEIWIP detectors can be utilized to engineer the $f_{0}$ in an n-type multilayer HEIWIP photodetector. However, the dopant migration can reduce the photoresponsivity, hence proper care should be taken during the fabrication of the n-type GaAs/AlGaAs multilayer HEIWIP to increase photoresponsivity.

\section{REFERENCES}

[1] P. V. V. Jayaweera, S. G. Matsik, A. G. U. Perera, Y. Paltiel, A. Sher, A. Raizman, H. Luo, and H. C. Liu, "GaSb homojunctions for farinfrared (terahertz) detection," Appl. Phys. Lett., vol. 90, no. 11, pp. 111 109-1-111 109-3, Mar. 2007.

[2] M. B. M. Rinzan, A. G. U. Perera, S. G. Matsik, H. C. Liu, Z. R. Wasilewski, and M. Buchanan, "AlGaAs emitter/GaAs barrier terahertz detector with a $2.3 \mathrm{THz}$ threshold," Appl. Phys. Lett., vol. 86, no. 7, p. 071112 , Feb. 2005.

[3] A. Weerasekara, M. Rinzan, S. Matsik, H. C. Liu, M. Buchanan, G. von Winckel, A. Stintz, S. Krishna, and A. G. U. Perera, "n-Type GaAs/AlGaAs heterostructure detector with a $3.2 \mathrm{THz}$ threshold frequency," Opt. Lett., vol. 32, no. 10, pp. 1335-1337, May 2007.

[4] D. H. Zhang, W. Shi, P. H. Zhang, and S. F. Yoon, "Doping effects on P-type InGaAs/AlGaAs quantum well structures for infrared photodetectors grown by molecular beam epitaxy," Jpn. J. Appl. Phys., vol. 38, no. 4A, pp. L360-L362, Apr. 1999.

[5] Y. Yamashita, A. Asano, Y. Nishioka, and H. Kobayashi, "Dependence of interface states in the Si band gap on oxide atomic density and interfacial 
roughness," Phys. Rev. B, Condens. Matter, vol. 59, no. 24, pp. $15872-$ 15 881, Jun. 1999.

[6] F. Capasso, A. Y. Cho, K. Mohammed, and P. W. Foy, "Doping interface dipoles: Tunable heterojunction barrier heights and band-edge discontinuities by molecular beam epitaxy," Appl. Phys. Lett., vol. 46, no. 7, pp. 664666, Apr. 1985

[7] J. S. Rimmer, B. Hamilton, P. Dawson, M. Missous, and A. R. Peaker, "Correlation between optical spectroscopy and capacitance-voltage profile simulation applied to interface states in multilayer GaAs/AlGaAs heterostructures," J. Appl. Phys., vol. 73, no. 10, pp. 5032-5037, May 1993.
[8] H. Kroemer, W.-Y. Chien, J. J. S. Harris, and D. D. Edwall, "Measurement of isotype heterojunction barriers by $C-V$ profiling," Appl. Phys. Lett., vol. 36, no. 4, pp. 295-297, Feb. 1980.

[9] W. Z. Shen and A. G. U. Perera, "Low-frequency noise and interface states in GaAs homojunction far-infrared detectors," IEEE Trans. Electron Devices, vol. 46, no. 4, pp. 811-814, Apr. 1999.

[10] H. Okumura, S. Misawa, S. Yoshida, and S. Gonda, "Determination of the conduction-band discontinuities of GaAs/ $\mathrm{Al}_{x} \mathrm{Ga}_{1-x}$ As interfaces by capacitance-voltage measurements," Appl. Phys. Lett., vol. 46, no. 4, pp. 377-379, Feb. 1985. 